\title{
Long-Term Oncological Outcome Comparison between Intermediate- and High-Dose Radioactive Iodine Ablation in Patients with Differentiated Thyroid Carcinoma: A Propensity Score Matching Study
}

\author{
Kwangsoon Kim (D), Ja Seong Bae, and Jeong Soo Kim \\ Department of Surgery, College of Medicine, The Catholic University of Korea, Seoul, Republic of Korea \\ Correspondence should be addressed to Jeong Soo Kim; btskim@catholic.ac.kr
}

Received 24 November 2020; Revised 9 February 2021; Accepted 17 February 2021; Published 24 February 2021

Academic Editor: Claudio Casella

Copyright (c) 2021 Kwangsoon Kim et al. This is an open access article distributed under the Creative Commons Attribution License, which permits unrestricted use, distribution, and reproduction in any medium, provided the original work is properly cited.

\begin{abstract}
Background. Radioactive iodine (RAI) ablation is recommended for most patients with differentiated thyroid carcinoma (DTC) after total thyroidectomy (TT). We aimed to compare long-term outcomes between intermediate-dose (100 mCi) and high-dose $(150 \mathrm{mCi}) \mathrm{RAI}$ ablation therapy in patients with DTC using propensity score matching analysis. Methods. This was a retrospective study of 1448 patients with DTC who underwent RAI ablation after TT. Propensity score matching was performed using the extent of operation, tumor size, extrathyroidal extension, multifocality, lymphatic invasion, vascular invasion, perineural invasion, number of positive lymph nodes (LNs), ATA risk stratification system, T stage, N stage, TNM stage, preoperative serum Tg and $\mathrm{TgAb}$ levels, and post-RAI serum $\mathrm{Tg}$ and $\mathrm{TgAb}$ levels. Results. Recurrence rates in the intermediate- and high-dose groups were $3.1 \%$ and $5.6 \%$, respectively. After propensity score matching, LN ratio $>0.22(\mathrm{HR}, 2.915 ; 95 \% \mathrm{CI}, 1.228-6.918 ; p=0.015)$ and serum $\mathrm{Tg}>10 \mathrm{ng} / \mathrm{mL}$ after RAI (HR, 3.976; 95\% CI, 1.839-8.595; $p<0.001$ ) were significant predictors of recurrence. Kaplan-Meier analysis showed no significant difference in DFS before or after propensity score matching $(p=0.074$ and $p=0.378$, respectively). Conclusions. Intermediate-dose RAI ablation for the adjuvant treatment of DTC is sufficient as compared to high-dose RAI ablation. Further prospective or multicenter studies should be conducted to clarify the prognosis of intermediate-dose RAI ablation.
\end{abstract}

\section{Introduction}

Differentiated thyroid carcinoma (DTC) is the most frequent endocrine malignancy. Its worldwide incidence has been increasing over the past several decades [1-3]. Papillary thyroid carcinoma (PTC) is the most common form of DTC and accounts for $90 \%$ of all thyroid malignancies, and the second most common form of DTC in Korea is follicular thyroid carcinoma (FTC) [4]. The development of diagnostic techniques and early screening has led to an increase in the diagnosis of papillary microcarcinoma $[5,6]$.

Management of DTC generally involves surgery followed by postoperative adjuvant therapy, including radioactive iodine (RAI) ablation and thyrotropin suppression [7]. The American Thyroid Association (ATA) management guidelines recommend a patient-individualized approach [8]. RAI ablation has been recommended for most DTC patients after total thyroidectomy (TT). RAI ablation has been recommended for most DTC patients after total thyroidectomy (TT). Its purpose is to eliminate normal remnant thyroid tissue to achieve undetectable serum thyroglobulin $(\mathrm{Tg})$ and to eradicate any foci of carcinoma to prevent recurrence and to perform a diagnostic whole-body scanning to detect persistent thyroid carcinoma [8]. More recently, RAI ablation is being reserved for selected patients with higher recurrence risk after surgery alone. Although RAI ablation is usually safe, well tolerated, and effective in 
these patients, the optimal dose to maximize treatment effect while minimizing side effects remains controversial.

The side effects include transient neck pain, nausea, vomiting, loss of taste, acute and/or chronic salivary gland dysfunction, sialadenitis, temporary gonadal dysfunction, and rarely radiation-related second malignancy [9-11]. Since most side effects are dose-dependent, it is important to use the minimal RAI dose that will achieve the maximal ablation effect for the patient's quality of life [12].

Many previous studies comparing oncologic outcomes after various RAI doses in DTC patients have reported different outcomes [13-17]. To the best of our knowledge, this is one of the largest case series to date comparing the long-term oncological outcomes between intermediate- and high-dose RAI ablation. Therefore, the objective of this study was to compare long-term outcomes between intermediateand high-dose RAI ablation therapy in patients with DTC using propensity score matching analysis in a large series of patients.

\section{Materials and Methods}

2.1. Patients. We retrospectively reviewed the medical records of 1539 patients with DTC who underwent RAI ablation after TT and/or modified radical neck dissection (mRND) from January 2009 to December 2014 at Seoul St. Mary's Hospital (Seoul, Korea). After excluding 18 and 73 patients due to inadequate data and loss of follow-up, respectively, 1448 patients were analyzed. Patients were categorized into two groups according to RAI dose, intermediate dose $(100 \mathrm{mCi})$, and high dose $(150 \mathrm{mCi})$. The lymph node (LN) ratio was defined as the number of positive LNs divided by the number of harvested LNs. The mean follow-up duration was 92.2 \pm 23.4 months (range, 62-134). This study was conducted in accordance with the Declaration of Helsinki (as revised in 2013). This study was approved by the institutional review board of Seoul St. Mary's Hospital, The Catholic University of Korea (IRB No: KC20RISI0278), which waived the requirement for informed consent due to the retrospective nature of this study.

2.2. Postoperative Management and Follow-Up. All patients were managed according to ATA management guidelines [8] after surgical treatment and received suppressive doses of levothyroxine. Regular follow-up consisted of physical examination, thyroid function and antithyroglobulin antibody level testing, and ultrasonography of the neck every 3-6 months and annually thereafter. Additional diagnostic imaging modalities, such as computed tomography and positron emission tomography/computed tomography, were performed as necessary to confirm the recurrent disease.

2.3. RAI Protocol. RAI ablation was performed 6-8 weeks after surgery by an experienced nuclear medicine physician. All study patients underwent thyroid hormone withdrawal for at least 4 weeks prior to RAI ablation or two daily injections of $0.9 \mathrm{mg}$ recombinant human thyroid-stimulating hormone (TSH) for TSH stimulation. Patients have also prescribed a low iodine diet before ablation. RAI was administered once the serum TSH level was $>30 \mathrm{mUI} / \mathrm{L}$ to enhance iodine uptake into the remnant tissue and allow the delivery of a higher radiation dose. The initial RAI dose was $100 \mathrm{mCi}$ (intermediate-dose) in 1146 patients and $150 \mathrm{mCi}$ (high-dose) in 302 patients. Radioiodine whole-body scintigraphy and single-proton emission computed tomography were performed 5-7 days after RAI ablation.

2.4. Laboratory Studies. Patients underwent venipuncture to collect a blood sample for measurement of serum $\mathrm{Tg}$ and serum $\mathrm{Tg}$ antibody (TgAb) levels. Serum $\mathrm{Tg}$ was measured using an immunoradiometric Tg assay (CIS Bio International, Saclay, France) with a functional sensitivity of $0.2 \mathrm{ng} /$ $\mathrm{mL}$. Serum $\mathrm{TgAb}$ concentration was measured using a radioimmunoassay (DIAsource, Rue du Bosquet, Belgium); serum $\mathrm{TgAb}$ level $<60 \mathrm{IU} / \mathrm{mL}$ was considered negative [18]. The $\mathrm{Tg}$ ratio was defined as post-RAI serum $\mathrm{Tg}$ level/preoperative serum $\mathrm{Tg}$ level.

2.5. Statistical Analysis. Continuous and quantitative data are reported as means with standard deviation, and categorical qualitative data are presented as numbers with percentages. The student's $t$-test was used to compare continuous variables; categorical variables were compared using Pearson's chi-square test or Fisher's exact test. Univariate and multivariate Cox regression analyses were performed to identify disease-free survival (DFS) predictors using calculated hazard ratios (HRs) with 95\% confidence intervals (CIs). Kaplan-Meier DFS curves were compared using the log-rank test. Receiver operating characteristics (ROC) curve analysis was performed to determine the optimal cutoff values for the $\mathrm{LN}$ ratio and $\mathrm{Tg}$ ratio.

To reduce the impact of selection bias and potential confounding factors, propensity score matching was performed using sixteen clinicopathological and biochemical characteristics: extent of operation, tumor size, extrathyroidal extension, multifocality, lymphatic invasion, vascular invasion, perineural invasion, number of positive LNs, ATA risk stratification system, T stage, $\mathrm{N}$ stage, TNM stage, preoperative serum $\mathrm{Tg}$ and $\mathrm{TgAb}$ levels, and post-RAI serum $\mathrm{Tg}$ and $\mathrm{TgAb}$ levels. Individual patient propensity scores were calculated using logistic regression analysis. After propensity score matching, the baseline clinicopathological and biochemical characteristics representative of long-term oncologic outcomes were compared between the two groups. DFS after propensity score matching was compared using Kaplan-Meier survival analysis with the log-rank test in the same way as before propensity score matching. $p<0.05$ was considered significant. All statistical analyses were performed using SPSS software version 24.0 for Windows (IBM Corp., Armonk, NY, USA).

\section{Results}

3.1. Comparison of Baseline Clinicopathological Characteristics between the Intermediate- and High-Dose Groups before and after Propensity Score Matching. The baseline 
clinicopathological characteristics of the intermediate- and high-dose groups are shown in Table 1. Mean age, gender, type of carcinoma, bilaterality, lymphatic invasion, vascular invasion, $\mathrm{BRAF}^{\mathrm{V} 600 \mathrm{E}}$ positivity, and the number of harvested LNs did not significantly differ between groups. A significantly greater proportion of patients in the high-dose group underwent more extensive surgery (TT and mRND) compared to the intermediate-dose group $(24.5 \%$ vs. $16.8 \%$, $p=0.003)$. Tumor size was significantly larger in the highdose group $(1.4 \pm 0.9 \mathrm{~cm}$ vs. $1.2 \pm 0.8 \mathrm{~cm}, p=0.001)$. The high-dose group had a significantly higher prevalence of extrathyroidal extension (ETE) but a significantly lower prevalence of multifocality $(19.2 \%$ vs. $8.1 \%, p<0.001$; and $44.0 \%$ vs. $53.8 \%, p=0.003$, respectively). Although the number of harvested LNs did not significantly differ between the groups, the number of positive LNs was significantly greater in the high-dose group $(5.2 \pm 6.0$ vs. $4.0 \pm 5.9 \mathrm{~cm}$, $p=0.004)$. In terms of ATA risk stratification, the high-dose group had a significantly higher risk of recurrence $(p<0.001)$. Patients in the high-dose group were diagnosed with significantly higher T stage, $\mathrm{N}$ stage, and TNM stage tumors $(p<0.001, p<0.001$, and $p=0.008$, respectively). Thirty-five (3.1\%) patients in the intermediate-dose group and $17(5.6 \%)$ patients in the high-dose group experienced recurrence; the difference was significant $(p=0.038)$.

Propensity score matching yielded 552 matched patient pairs. There were no differences in baseline clinicopathological characteristics between the matched groups (Table 1).

\subsection{Comparison of Perioperative Biochemical Characteristics} between the Intermediate- and High-Dose Groups before and after Propensity Score Matching. Table 2 shows the perioperative biochemical characteristics of the intermediateand high-dose groups. Preoperative TSH level, preoperative serum $\mathrm{Tg}$ level, the ratio of preoperative serum $\mathrm{Tg}$ of $1 \mathrm{ng} /$ $\mathrm{mL}$, the ratio of positivity of preoperative serum $\operatorname{TgAb}$, and the ratio of positivity of serum $\mathrm{TgAb}$ after RAI did not significantly differ between the intermediate- and high-dose groups. However, preoperative serum $\mathrm{Tg}>10 \mathrm{ng} / \mathrm{mL}$ was significantly higher in the high-dose group (63.9\% vs. $55.9 \%$, $p=0.013)$. The high-dose group had a significantly higher ratio of serum $\mathrm{Tg}>1 \mathrm{ng} / \mathrm{mL}$ and $>10 \mathrm{ng} / \mathrm{mL}$ after RAI $(49.0 \%$ vs. $42.0 \%, p=0.031$; and $18.5 \%$ vs. $8.6 \%, p<0.001$, respectively). After propensity score matching, there were no differences in perioperative biochemical characteristics between the groups (Table 2).

\subsection{Univariate and Multivariate Analyses of Recurrence Risk} Factors before and after Propensity Score Matching. Table 3 presents the results of univariate and multivariate Cox regression analyses before propensity score matching. Perineural invasion (HR, 2.918; 95\% CI, 1.147-5.217; $p=0.025$ ), number of positive LNs (HR, 1.039; 95\% CI, $0.109-1.059 ; p<0.001)$, LN ratio $>0.22$ (HR, 2.373; 95\% CI, $1.260-4.469 ; p=0.007)$, and positive serum $\mathrm{TgAb}$ after RAI (HR, 2.564; 95\% CI, 1.197-5.508; $p=0.015$ ) were significant predictors of recurrence. The most significant predictor of recurrence was serum $\mathrm{Tg}>10 \mathrm{ng} / \mathrm{mL}$ after RAI (HR, 4.504;
95\% CI, 2.521-8.045; $p<0.001)$. After propensity score matching, LN ratio $>0.22$ (HR, 2.915; 95\% CI, 1.228-6.918; $p=0.015)$ and serum $\mathrm{Tg}>10 \mathrm{ng} / \mathrm{mL}$ after RAI (HR, 3.976; 95\% CI, 1.839-8.595; $p<0.001$ ) were confirmed as significant predictors of recurrence (Table 4).

In the Kaplan-Meier analysis, DFS did not significantly differ between the intermediate- and high-dose groups before or after propensity score matching $(p=0.074$ and $p=0.378$, respectively; Figures 1 and 2). After propensity score matching, multivariate Cox regression analysis identified $\mathrm{LN}$ ratio $>0.22$ and serum $\mathrm{Tg}>10 \mathrm{ng} / \mathrm{mL}$ after RAI as significant predictors of recurrence. DFS according to LN ratio and serum $\mathrm{Tg}$ after RAI was significantly different between the two groups as well $(p=0.005$ and $p<0.001$, respectively; Figures 3 and 4).

3.4. Subgroup Analysis of the Intermediate- and High-Dose Groups according to ATA Risk Stratification after Propensity Score Matching. For subgroup outcome analysis according to ATA risk stratification, the patients were divided into intermediate-risk $(n=390)$ and high-risk $(n=84)$ groups. In the intermediate-risk group, there was no significant difference in recurrence between the intermediate- and highdose patients $(6.0 \%$ vs $5.9 \%, p=0.979)$. In the high-risk group, there was no significant difference in recurrence according to dose as well ( $4.4 \%$ vs $2.6 \%, p=0.643$ ) (Table 5).

\section{Discussion}

The management of thyroid carcinoma is individualized and must take into account risk factors for death and recurrence. After RAI was first proposed for therapeutic use in patients with hyperthyroidism by researchers at the Massachusetts General Hospital [19], it has been widely used in patients with DTC and is considered a first-line adjuvant therapy after TT [8].

RAI ablation is not routinely recommended after TT for patients with unifocal and intrathyroidal papillary microcarcinoma without other high-risk factors [20,21]. However, RAI ablation should be considered in ATA intermediate-risk patients [22] and is routinely recommended for those with high risk $[8,23]$. Thus, postoperative ATA risk classification plays an important role in determining the use of RAI ablation. Additional considerations may include patient comorbidities, patient preferences, and preferred disease surveillance procedures [14].

Numerous studies have examined the effect of RAI ablation on recurrence and mortality in patients with DTC. Mazzaferri et al. reported that RAI ablation significantly reduced both recurrence and mortality [24], and Sawka et al. reported that RAI ablation significantly reduced recurrence and distant metastasis. As a result of these studies, RAI ablation became the standard treatment for patients with DTC after TT [25]. However, Jonklaas et al. found no survival benefit after RAI ablation in TNM stage I DTC patients [26], and Schvartz et al. found that RAI ablation in ATA low-risk DTC patients had no impact on recurrence or mortality [27]. Thus, RAI ablation remains controversial in ATA low-risk DTC patients. In 2015, 
TABle 1: Comparison of baseline clinicopathological characteristics between intermediate- (100 mCi) and high-dose (150 mCi) groups before and after propensity score matching.

\begin{tabular}{|c|c|c|c|c|c|c|}
\hline & \multicolumn{2}{|c|}{ Before matching } & \multirow{2}{*}{$p$ value } & \multicolumn{2}{|c|}{ After matching } & \multirow{2}{*}{$\underset{\text { value }}{p}$} \\
\hline & $100 \mathrm{mCi}(n=1146)$ & $150 \mathrm{mCi}(n=302)$ & & $100 \mathrm{mCi}(n=276)$ & $150 \mathrm{mCi}(n=276)$ & \\
\hline Age (years) & $\begin{array}{c}46.3 \pm 12.2 \text { (range, } \\
11-81)\end{array}$ & $\begin{array}{c}45.9 \pm 12.7 \text { (range } \\
18-74)\end{array}$ & 0.599 & $\begin{array}{c}45.6 \pm 13.3 \text { (range, } \\
12-81 \text { ) }\end{array}$ & $\begin{array}{c}45.6 \pm 12.8 \text { (range, } \\
18-74)\end{array}$ & 0.997 \\
\hline$\geq 55$ & $294(25.7 \%)$ & $84(27.8 \%)$ & 0.462 & $201(72.8 \%)$ & $202(73.2 \%)$ & 0.924 \\
\hline$<55$ & $852(74.3 \%)$ & $218(72.2 \%)$ & & $75(27.2 \%)$ & $74(26.8 \%)$ & \\
\hline Female & $869(75.8 \%)$ & $238(78.8 \%)$ & 0.287 & $206(74.6 \%)$ & $214(77.5 \%)$ & 0.485 \\
\hline Type of carcinoma & & & 0.185 & & & 1.000 \\
\hline PTC & 1137 (99.2\%) & 297 (98.3\%) & & $272(98.6 \%)$ & $272(98.6 \%)$ & \\
\hline FTC & $9(0.8 \%)$ & $5(1.7 \%)$ & & $4(1.4 \%)$ & $4(1.4 \%)$ & \\
\hline Extent of operation & & & 0.003 & & & 0.616 \\
\hline TT & $953(83.2 \%)$ & $228(75.5 \%)$ & & $208(75.4 \%)$ & $214(77.5 \%)$ & \\
\hline TT and mRND & $193(16.8 \%)$ & $74(24.5 \%)$ & & $68(24.6 \%)$ & $62(22.5 \%)$ & \\
\hline Tumor size $(\mathrm{cm})$ & $\begin{array}{c}1.2 \pm 0.8 \text { (range } \\
0.2-9.0 \text { ) }\end{array}$ & $\begin{array}{c}1.4 \pm 0.9 \text { (range } \\
0.2-6.5 \text { ) }\end{array}$ & 0.001 & $\begin{array}{c}1.4 \pm 0.8 \text { (range } \\
0.2-5.4 \text { ) }\end{array}$ & $\begin{array}{c}1.3 \pm 0.8 \text { (range } \\
0.2-6.5 \text { ) }\end{array}$ & 0.513 \\
\hline ETE & $93(8.1 \%)$ & $58(19.2 \%)$ & $<0.001$ & $48(17.4 \%)$ & $40(14.5 \%)$ & 0.416 \\
\hline Multifocality & $617(53.8 \%)$ & $133(44.0 \%)$ & 0.003 & $133(48.2 \%)$ & $127(46.0 \%)$ & 0.670 \\
\hline Bilaterality & $416(36.3 \%)$ & $99(32.8 \%)$ & 0.280 & $85(30.8 \%)$ & $94(34.1 \%)$ & 0.467 \\
\hline Lymphatic invasion & $491(42.8 \%)$ & $147(48.7 \%)$ & 0.078 & $139(50.4 \%)$ & $128(46.4 \%)$ & 0.394 \\
\hline Vascular invasion & $38(3.3 \%)$ & $17(5.6 \%)$ & 0.088 & $15(5.4 \%)$ & $15(5.4 \%)$ & 1.000 \\
\hline Perineural invasion & $28(2.4 \%)$ & $19(6.3 \%)$ & 0.002 & $12(4.3 \%)$ & $10(3.6 \%)$ & 0.828 \\
\hline $\mathrm{BRAF}^{\mathrm{V} 600 \mathrm{E}}$ positive & $700 / 880(79.5 \%)$ & $126 / 158(79.7 \%)$ & 0.954 & $173 / 216(80.1 \%)$ & $111 / 140(79.3 \%)$ & 0.893 \\
\hline Harvested LNs & $19.6 \pm 21.6$ & $20.4 \pm 21.7$ & 0.554 & $22.9 \pm 23.4$ & $19.5 \pm 21.3$ & 0.073 \\
\hline Positive LNs & $4.0 \pm 5.9$ & $5.2 \pm 6.0$ & 0.004 & $4.9 \pm 5.7$ & $4.9 \pm 5.9$ & 0.901 \\
\hline $\begin{array}{l}\text { ATA risk } \\
\text { stratification }\end{array}$ & & & $<0.001$ & & & 0.740 \\
\hline Low & $273(23.8 \%)$ & 48 (15.9\%) & & 47 (17.0\%) & $51(18.5 \%)$ & \\
\hline Intermediate & $787(68.7 \%)$ & $197(65.2 \%)$ & & $184(66.7 \%)$ & $186(67.4 \%)$ & \\
\hline High & $86(7.5 \%)$ & 57 (18.9\%) & & $45(16.3 \%)$ & $39(14.1 \%)$ & \\
\hline T stage & & & $<0.001$ & & & 0.773 \\
\hline $\mathrm{T} 1$ & $953(83.2 \%)$ & $215(71.2 \%)$ & & $200(72.5 \%)$ & $208(75.4 \%)$ & \\
\hline $\mathrm{T} 2$ & $85(7.4 \%)$ & $27(8.9 \%)$ & & $27(9.8 \%)$ & $26(9.4 \%)$ & \\
\hline $\mathrm{T} 3 \mathrm{a}$ & $15(1.3 \%)$ & $2(0.7 \%)$ & & $1(0.4 \%)$ & $2(0.7 \%)$ & \\
\hline $\mathrm{T} 3 \mathrm{~b}$ & $90(7.9 \%)$ & $55(18.2 \%)$ & & $47(17.0 \%)$ & $38(13.8 \%)$ & \\
\hline $\mathrm{T} 4$ & $3(0.3 \%)$ & $3(1.0 \%)$ & & $1(0.4 \%)$ & $2(0.7 \%)$ & \\
\hline $\mathrm{N}$ stage & & & $<0.001$ & & & 0.820 \\
\hline No & $284(24.8 \%)$ & $52(17.2 \%)$ & & 47 (17.0\%) & $50(18.1 \%)$ & \\
\hline N1a & $669(58.4 \%)$ & $176(58.3 \%)$ & & $161(58.3 \%)$ & $164(59.4 \%)$ & \\
\hline N1b & $193(16.8 \%)$ & $74(24.5 \%)$ & & $68(24.6 \%)$ & $62(22.5 \%)$ & \\
\hline TNM stage & & & 0.008 & & & 0.608 \\
\hline Stage I & $939(81.9 \%)$ & $228(75.5 \%)$ & & $218(79.0 \%)$ & $212(76.8 \%)$ & \\
\hline Stage II & $207(18.1 \%)$ & $73(24.2 \%)$ & & $58(21.0 \%)$ & $64(23.2 \%)$ & 0.694 \\
\hline Stage III & $0(0 \%)$ & $1(0.3 \%)$ & & $0(0 \%)$ & $0(0 \%)$ & \\
\hline Recurrence & $35(3.1 \%)$ & $17(5.6 \%)$ & 0.038 & $15(5.4 \%)$ & $12(4.3 \%)$ & \\
\hline
\end{tabular}

Data are expressed as the patient's number (\%) or mean \pm SD. A statistically significant difference was defined as $p<0.05$. Abbreviation: PTC, papillary thyroid carcinoma; FTC, follicular thyroid carcinoma; TT, total thyroidectomy; mRND, modified radical neck dissection; ETE, extrathyroidal extension; LN, lymph node; ATA, American thyroid association; T, tumor; N, node; M, metastasis.

the ATA recommended using RAI ablation in DTC patients according to recurrence risk [8].

This study compared long-term outcomes between intermediate- and high-dose RAI ablation using recurrence and DFS as measures. The high-dose group was significantly associated with more aggressive tumor characteristics, including larger tumor size, higher prevalence of ETE, higher number of positive LNs, higher ATA risk classification, higher TNM stage, and higher preoperative and postablation serum Tg level. Therefore, confounding and selection bias may have been introduced. To minimize their effects, propensity score matching analysis was performed to adjust for several clinicopathological characteristics.

Although the recurrence rate was significantly higher in the high-dose group before propensity score matching $(3.1 \%$ vs. $5.6 \%, p=0.038)$, after matching, the recurrence rates were similar $(5.4 \%$ vs. $4.3 \%, p=0.694)$, and there was no significant difference in DFS (log-rank $p=0.378$ ). Only LN ratio $>0.22(\mathrm{HR}, 2.915, p=0.015)$ and serum $\mathrm{Tg}$ level 
TABLE 2: Comparison of perioperative biochemical characteristics between intermediate- (100 mCi) and high-dose (150 mCi) groups before and after propensity score matching.

\begin{tabular}{|c|c|c|c|c|c|c|}
\hline & \multicolumn{2}{|c|}{ Before matching } & \multirow{2}{*}{$p$ value } & \multicolumn{2}{|c|}{ After matching } & \multirow{2}{*}{$p$ value } \\
\hline & $100 \mathrm{mCi}(n=1146)$ & $150 \mathrm{mCi}(n=302)$ & & $100 \mathrm{mCi}(n=1146)$ & $150 \mathrm{mCi}(n=302)$ & \\
\hline Pre-op. TSH (mIU/L) & $2.9 \pm 10.9$ & $2.2 \pm 1.8$ & 0.276 & $2.1 \pm 1.5$ & $2.2 \pm 1.8$ & 0.549 \\
\hline Pre-op. serum $\operatorname{Tg}(\mathrm{ng} / \mathrm{mL})$ & $30.9 \pm 75.6$ & $39.7 \pm 89.4$ & 0.086 & $38.7 \pm 99.9$ & $39.9 \pm 92.4$ & 0.885 \\
\hline$\leq 1$ & $114(9.9 \%)$ & $30(9.9 \%)$ & 1.000 & $30(10.9 \%)$ & $28(10.1 \%)$ & 0.890 \\
\hline$>1$ & $1032(90.1 \%)$ & $272(90.1 \%)$ & & $246(89.1 \%)$ & $248(89.9 \%)$ & \\
\hline$\leq 10$ & $505(44.1 \%)$ & $109(36.1 \%)$ & 0.013 & $111(40.2 \%)$ & $99(35.9 \%)$ & 0.335 \\
\hline$>10$ & $641(55.9 \%)$ & $193(63.9 \%)$ & & $165(59.8 \%)$ & $177(64.1 \%)$ & \\
\hline Pre-op. serum $\operatorname{TgAb}(\mathrm{IU} / \mathrm{mL})$ & $113.7 \pm 595.9$ & $207.0 \pm 884.2$ & 0.031 & $170.5 \pm 889.8$ & $144.3 \pm 569.0$ & 0.681 \\
\hline Negative & $922(80.5 \%)$ & $235(77.8 \%)$ & 0.333 & $225(81.5 \%)$ & $216(78.3 \%)$ & 0.396 \\
\hline Positive & $224(19.5 \%)$ & $67(22.2 \%)$ & & $51(18.5 \%)$ & $60(21.7 \%)$ & \\
\hline Post-RAI serum $\mathrm{Tg}(\mathrm{ng} / \mathrm{mL})$ & $3.8 \pm 12.5$ & $9.8 \pm 41.2$ & $<0.001$ & $5.3 \pm 13.7$ & $6.7 \pm 15.9$ & 0.263 \\
\hline$\leq 1$ & $665(58.0 \%)$ & $154(51.0 \%)$ & 0.031 & $141(51.1 \%)$ & $144(52.2 \%)$ & 0.865 \\
\hline$>1$ & $481(42.0 \%)$ & $148(49.0 \%)$ & & $135(48.9 \%)$ & $132(47.8 \%)$ & \\
\hline$\leq 10$ & $1048(91.4 \%)$ & $246(81.5 \%)$ & $<0.001$ & $245(88.8 \%)$ & $229(83.0 \%)$ & 0.066 \\
\hline$>10$ & $98(8.6 \%)$ & $56(18.5 \%)$ & & $31(11.2 \%)$ & $47(17.0 \%)$ & \\
\hline Post-RAI serum $\operatorname{TgAb}(\mathrm{IU} / \mathrm{mL})$ & $31.1 \pm 100.9$ & $64.2 \pm 324.8$ & 0.003 & $44.5 \pm 163.9$ & $35.6 \pm 133.3$ & 0.484 \\
\hline Negative & $1067(93.1 \%)$ & $272(90.1 \%)$ & 0.086 & $252(91.3 \%)$ & $252(91.3 \%)$ & 1.000 \\
\hline Positive & $79(6.9 \%)$ & $31(9.9 \%)$ & & $24(8.7 \%)$ & $241(8.7 \%)$ & \\
\hline
\end{tabular}

Data are expressed as the patient's number (\%) or mean \pm SD. A statistically significant difference was defined as $p<0.05 . \mathrm{Negative} \mathrm{TgAb}, \mathrm{TgAb}<60 \mathrm{IU} / \mathrm{mL}$; positive $\mathrm{TgAb}, \mathrm{TgAb} \geq 60 \mathrm{IU} / \mathrm{mL}$. Abbreviation: $\mathrm{TSH}$, thyroid-stimulating hormone; pre-op, preoperative; post-RAI, after radioactive iodine therapy; $\mathrm{Tg}$, thyroglobulin; TgAb, thyroglobulin antibody.

TABLE 3: Univariate and multivariate analyses of recurrence risk factors before propensity score matching.

\begin{tabular}{|c|c|c|c|c|}
\hline & \multicolumn{2}{|c|}{ Univariate } & \multicolumn{2}{|c|}{ Multivariate } \\
\hline & HR $(95 \%$ CI $)$ & $p$ value & HR $(95 \% \mathrm{CI})$ & $p$ value \\
\hline Age & $0.962(0.940-0.984)$ & 0.001 & & \\
\hline \multicolumn{5}{|l|}{ Extent of operation } \\
\hline $\mathrm{TT}$ & Ref. & & & \\
\hline TT and mRND & $2.009(1.114-3.621)$ & 0.020 & & \\
\hline Tumor size & $1.382(1.119-1.707)$ & 0.003 & & \\
\hline ETE & $2.629(1.379-5.013)$ & 0.003 & & \\
\hline Lymphatic invasion & $2.734(1.531-4.884)$ & 0.001 & & \\
\hline Vascular invasion & $4.114(1.855-9.123)$ & 0.001 & & \\
\hline Perineural invasion & $3.185(1.267-8.010)$ & 0.014 & $2.918(1.147-5.217)$ & 0.025 \\
\hline Harvested LNs & $1.017(1.008-1.025)$ & $<0.001$ & & \\
\hline Positive LNs & $1.062(1.045-1.080)$ & $<0.001$ & 1.039 (1.019-1.059) & $<0.001$ \\
\hline \multicolumn{5}{|l|}{$\mathrm{LN}$ ratio } \\
\hline$\leq 0.22$ & Ref. & & Ref. & \\
\hline$>0.22$ & $3.513(1.928-6.401)$ & $<0.001$ & $2.373(1.260-4.469)$ & 0.007 \\
\hline \multicolumn{5}{|l|}{ ATA risk stratification } \\
\hline Low & Ref. & & & \\
\hline Intermediate & $4.348(1.344-14.069)$ & 0.014 & & \\
\hline High & $7.734(2.128-28.104)$ & 0.002 & & \\
\hline \multicolumn{5}{|l|}{ T stage } \\
\hline $\mathrm{T} 3 \mathrm{~b}$ & $2.919(1.511-5.637)$ & 0.001 & & \\
\hline \multicolumn{5}{|l|}{$\mathrm{N}$ stage } \\
\hline No & Ref. & & & \\
\hline N1a & $2.589(1.006-6.662)$ & 0.048 & & \\
\hline N1b & $4.264(1.561-11.650)$ & 0.005 & & \\
\hline Pre-op. serum Tg & $1.003(1.002-1.005)$ & $<0.001$ & & \\
\hline$\leq 10 \mathrm{ng} / \mathrm{mL}$ & Ref. & & & \\
\hline$>10 \mathrm{ng} / \mathrm{mL}$ & $2.242(1.197-4.201)$ & 0.012 & & \\
\hline Post-RAI serum $\mathrm{Tg}$ & $1.005(1.002-1.008)$ & 0.001 & & \\
\hline$\leq 1 \mathrm{ng} / \mathrm{mL}$ & Ref. & & & \\
\hline$>1 \mathrm{ng} / \mathrm{mL}$ & $3.257(1.788-5.934)$ & $<0.001$ & & \\
\hline$\leq 10 \mathrm{ng} / \mathrm{mL}$ & Ref. & & Ref. & \\
\hline
\end{tabular}


TABle 3: Continued.

\begin{tabular}{|c|c|c|c|c|}
\hline & \multicolumn{2}{|c|}{ Univariate } & \multicolumn{2}{|c|}{ Multivariate } \\
\hline & HR (95\% CI) & $p$ value & HR (95\% CI) & $p$ value \\
\hline$>10 \mathrm{ng} / \mathrm{mL}$ & $5.976(3.434-10.401)$ & $<0.001$ & $4.504(2.521-8.045)$ & $<0.001$ \\
\hline \multicolumn{5}{|c|}{ Post-RAI serum $\mathrm{TgAb}$} \\
\hline Negative & Ref. & & Ref. & \\
\hline Positive & $2.271(1.069-4.825)$ & 0.033 & $2.567(1.197-5.508)$ & 0.015 \\
\hline \multicolumn{5}{|l|}{ Tg. ratio } \\
\hline$\leq 0.077$ & Ref. & & & \\
\hline$>0.077$ & $2.264(1.256-4.079)$ & 0.007 & & \\
\hline
\end{tabular}

TABLE 4: Univariate and multivariate analyses of recurrence risk factors after propensity score matching.

\begin{tabular}{|c|c|c|c|c|}
\hline & \multicolumn{2}{|c|}{ Univariate } & \multicolumn{2}{|c|}{ Multivariate } \\
\hline & $\mathrm{HR}(95 \% \mathrm{CI})$ & $p$ value & $\operatorname{HR}(95 \% \mathrm{CI})$ & $p$ value \\
\hline Age & $0.960(0.931-0.990)$ & 0.009 & & \\
\hline Lymphatic invasion & $2.645(1.158-6.043)$ & 0.021 & & \\
\hline Positive LNs & $1.068(1.024-1.114)$ & 0.002 & & \\
\hline \multicolumn{5}{|l|}{$\mathrm{LN}$ ratio } \\
\hline$\leq 0.22$ & Ref. & & Ref. & \\
\hline$>0.22$ & $3.222(1.362-7.622)$ & 0.008 & $2.915(1.228-6.918)$ & 0.015 \\
\hline Pre-op. serum Tg & $1.002(1.000-1.004)$ & 0.046 & & \\
\hline$\leq 10 \mathrm{ng} / \mathrm{mL}$ & Ref. & & & \\
\hline$>10 \mathrm{ng} / \mathrm{mL}$ & $3.652(1.263-10.560)$ & 0.017 & & \\
\hline Post-RAI serum $\mathrm{Tg}$ & $1.018(1.005-1.031)$ & 0.006 & & \\
\hline$\leq 1 \mathrm{ng} / \mathrm{mL}$ & Ref. & & & \\
\hline$>1 \mathrm{ng} / \mathrm{mL}$ & $3.125(1.321-7.391)$ & 0.009 & & \\
\hline$\leq 10 \mathrm{ng} / \mathrm{mL}$ & Ref. & & Ref. & \\
\hline$>10 \mathrm{ng} / \mathrm{mL}$ & $4.385(2.034-9.451)$ & $<0.001$ & $3.976(1.839-8.595)$ & $<0.001$ \\
\hline
\end{tabular}

Data are expressed as hazard ratio (HR) and $95 \%$ confidence interval (CI). A $p$ value $<0.05$ was considered statistically significant. LN ratio is defined as the number of positive LNs/the number of harvested LNs. Tg ratio is defined as post-RAI Tg/preop Tg. Abbreviation: TT, total thyroidectomy; mRND, modified radical neck dissection; ETE, extrathyroidal extension; LN, lymph node; T, tumor; N, node; ATA, American thyroid association; pre-op, preoperative; postRAI, after radioactive iodine therapy; $\mathrm{Tg}$, thyroglobulin; $\mathrm{TgAb}$, thyroglobulin antibody.

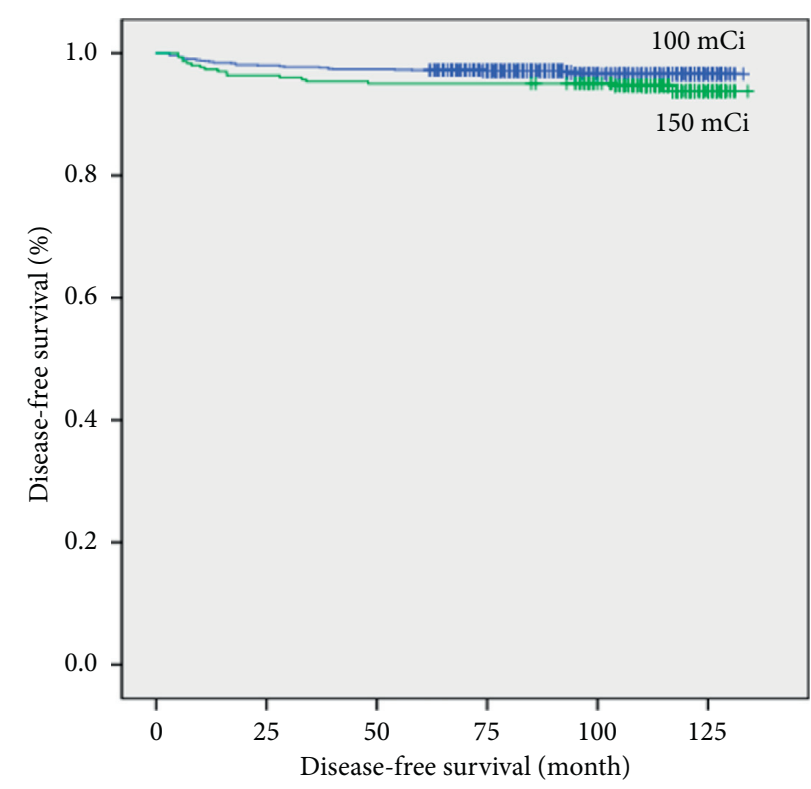

FIgURE 1: Disease-free survival curves of intermediate-dose $(100 \mathrm{mCi})$ and high-dose $(150 \mathrm{mCi})$ groups before propensity score matching $(p=0.074)$. 


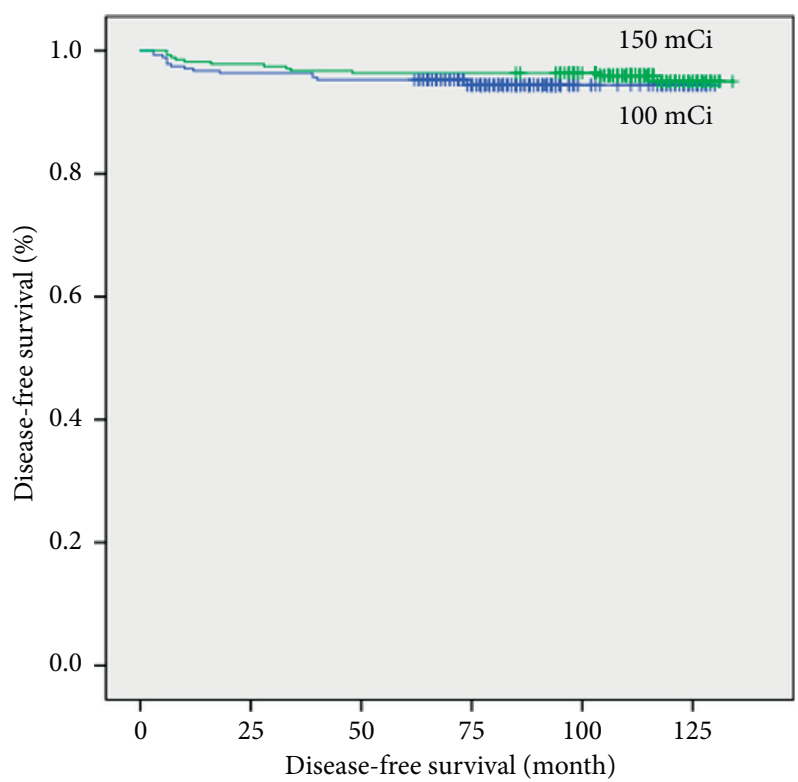

FIgURE 2: Disease-free survival curves of intermediate-dose $(100 \mathrm{mCi})$ and high-dose $(150 \mathrm{mCi})$ groups after propensity score matching $(p=0.378)$.

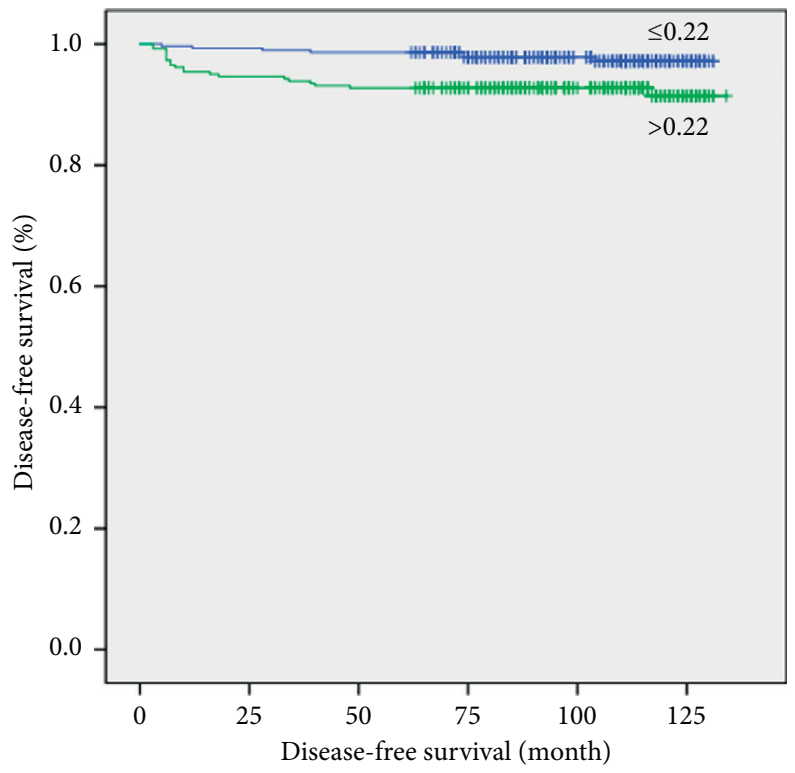

FIgURE 3: Disease-free survival curves according to post-RAI serum Tg level after propensity score matching (cut-off, $10 \mathrm{ng} / \mathrm{mL} ; p<0.001)$.

$>10 \mathrm{ng} / \mathrm{mL}$ after RAI $(\mathrm{HR}, 3.976, p<0.001)$ were identified as significant recurrence risk factors in the multivariate analysis, not RAI dose. Several studies have reported that high-dose RAI ablation has no major advantage over lowdose $[13,28,29]$. A meta-analysis by Cheng et al. validated that low-dose RAI was sufficient for thyroid remnant ablation as compared to high-dose with similar quality of life, less common side effects, and a shorter hospital stay [30]. However, to the best of our knowledge, this is the first study to compare long-term oncologic outcomes between intermediate- and high-dose RAI ablation using propensity score matching analysis.
Sacks et al. reported that low-risk patients achieve no survival or DFS benefit from RAI ablation [31]. This study included patients with intermediate- and high-risk ATA classifications in the subgroup analysis and confirmed that RAI dose did not affect recurrence in either $(6.0 \%$ vs $5.9 \%$, $p=0.979 ; 4.4 \%$ vs $2.6 \%, p=0.643$, respectively).

RAI ablation-related side effects can be divided into those related to ablation preparation and those related to radiation. In preparation for effective RAI ablation, levothyroxine should be discontinued to increase serum TSH. A hypothyroidism state may then result and manifest with various symptoms, such as weight gain, fatigue, cold intolerance, hypothermia, muscle 


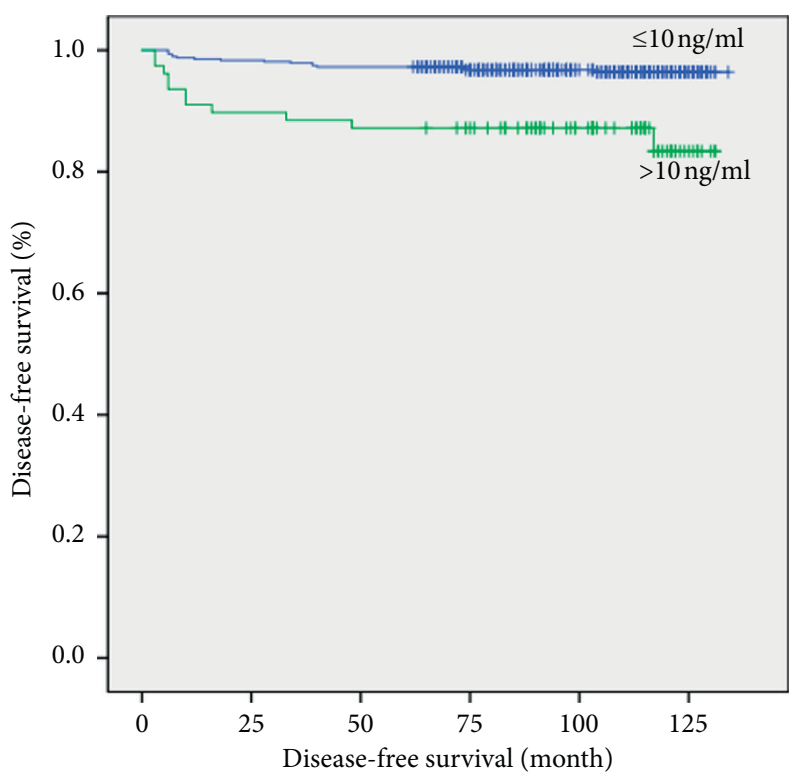

Figure 4: Disease-free survival curves according to lymph node ratio after propensity score matching (cut-off, $0.22 ; p=0.005$ ).

TABLE 5: Subgroup analysis between intermediate- $(100 \mathrm{mCi})$ and high-dose $(150 \mathrm{mCi})$ groups according to the ATA risk stratification after propensity score matching.

\begin{tabular}{|c|c|c|c|}
\hline Intermediate-risk & $100 m C i(n=184)$ & $150 m C i(n=186)$ & p value \\
\hline Age (years) & $45.4 \pm 14.1$ (range, $20-65)$ & $45.3 \pm 13.3($ range, $16-80)$ & 0.986 \\
\hline Female & $135(73.4 \%)$ & $141(75.8 \%)$ & 0.643 \\
\hline Tumor size $(\mathrm{cm})$ & $1.4 \pm 0.9$ & $1.4 \pm 0.9$ & 0.739 \\
\hline T stage & & & 0.840 \\
\hline $\mathrm{T} 1 / \mathrm{T} 2 / \mathrm{T} 3 \mathrm{a} / \mathrm{T} 3 \mathrm{~b} / \mathrm{T} 4$ & $157(85.2 \%) / 23(12.5 \%) / 1(0.6 \%) / 2(1.1 \%) / 1(0.6 \%)$ & $162(87.1 \%) / 22(11.8 \%) / 1(0.5 \%) / 1(0.5 \%) / 0$ & \\
\hline $\mathrm{N}$ stage & & & 0.577 \\
\hline N0/N1a/N1b & $37(20.1 \%) / 101(54.9 \%) / 46(25.0 \%)$ & $32(17.2 \%) / 112(60.2 \%) / 42(22.6 \%)$ & \\
\hline Pre-op. serum $\mathrm{Tg}(\mathrm{ng} / \mathrm{mL})$ & $44.3 \pm 113.1$ & $41.2 \pm 96.6$ & 0.774 \\
\hline Post-RAI serum $\mathrm{Tg}(\mathrm{ng} / \mathrm{mL})$ & $5.3 \pm 14.5$ & $7.7 \pm 17.3$ & 0.137 \\
\hline Recurrence & $11(6.0 \%)$ & $11(5.9 \%)$ & 0.979 \\
\hline High-risk & $100 m C i(n=45)$ & $150 m C i(n=39)$ & $p$ value \\
\hline Age (years) & $47.6 \pm 11.7($ range, $12-78)$ & $50.2 \pm 12.4($ range, $14-84)$ & 0.338 \\
\hline Female & $37(72.9 \%)$ & $37(78.5 \%)$ & 0.097 \\
\hline Tumor size $(\mathrm{cm})$ & $1.6 \pm 0.8$ & $1.4 \pm 0.6$ & 0.068 \\
\hline T stage & & & 0.213 \\
\hline $\mathrm{T} 1 / \mathrm{T} 2 / \mathrm{T} 3 \mathrm{a} / \mathrm{T} 3 \mathrm{~b} / \mathrm{T} 4$ & $0 / 0 / 0 / 45(100 \%) / 0$ & $0 / 0 / 0 / 37(94.9 \%) / 2(5.1 \%)$ & \\
\hline $\mathrm{N}$ stage & & & 0.114 \\
\hline N0/N1a/N1b & $4(8.9 \%) / 23(51.1 \%) / 18(40 \%)$ & $10(25.6 \%) / 15(38.5 \%) / 14(35.9 \%)$ & \\
\hline Pre-op. serum Tg (ng/mL) & $23.6 \pm 31.0$ & $53.3 \pm 118.7$ & 0.109 \\
\hline Post-RAI serum $\mathrm{Tg}(\mathrm{ng} / \mathrm{mL})$ & $4.3 \pm 10.0$ & $3.4 \pm 5.9$ & 0.612 \\
\hline Recurrence & $2(4.4 \%)$ & $1(2.6 \%)$ & 0.643 \\
\hline
\end{tabular}

Data are expressed as the patient's number (\%) or mean \pm SD. A statistically significant difference was defined as $p<0.05$. Abbreviation: ATA, American thyroid association; $\mathrm{T}$, tumor; $\mathrm{N}$, node; pre-op, preoperative; post-RAI, after radioactive iodine therapy; $\mathrm{Tg}$, thyroglobulin.

cramps, and constipation. Alternatively, in low- or intermediate-risk patients, recombinant human TSH can be administered instead of discontinuing levothyroxine [8,32]. Although RAI ablation is safe, radiation-related side effects are dosedependent [12] and can occur even at relatively low doses $[6,33]$. These include salivary gland dysfunction, temporary gonadal dysfunction, and secondary malignancy [34-36]. Higher RAI doses can lower the patient's quality of life without providing treatment benefits. This study suggests that a lower
RAI dose can achieve an equivalent treatment effect with fewer side effects.

This study has several limitations. Its retrospective single-center design may have introduced selection bias. However, propensity score matching was performed to adjust for differences in clinicopathological characteristics and minimize bias. In addition, we included patients of all ATA risk classifications, from low to high risk. Although we performed a subgroup analysis of 
intermediate- and high-risk patients, this may be a limitation. Moreover, we did not evaluate side effects related to RAI ablation, which are dose-dependent. Although an examination of side effects would have been beneficial, their objective evaluation is difficult as most side effects are subjective in nature. Finally, the follow-up period of this study was relatively short, which limited the ability to compare long-term oncological outcomes between the intermediate- and high-dose groups. Future studies evaluating prognostic factors in patients with DTC will require longer follow-up because of the indolent nature of the disease. Nonetheless, this study's strength is its follow-up of every patient and use of a standardized laboratory and imaging protocol in a single institution.

\section{Conclusions}

Intermediate-dose RAI ablation for the adjuvant treatment of DTC is sufficient as compared to high-dose RAI ablation. To the best of our knowledge, this is the first study to compare the long-term oncological outcome between intermediate- and high-dose RAI ablation. Further prospective or multicenter studies are warranted to clarify the prognosis of intermediatedose RAI ablation.

\section{Data Availability}

The data that support the findings of this study are available on request from the corresponding author. The data are not publicly available due to privacy or ethical restrictions.

\section{Conflicts of Interest}

The authors declare that there are no conflicts of interest.

\section{Acknowledgments}

This research was supported by the Basic Science Research Program through the National Research Foundation of Korea (NRF) funded by the Ministry of Education (NRF2015R1D1A1A01058798).

\section{References}

[1] J. D. Cramer, P. Fu, K. C. Harth, S. Margevicius, and S. M. Wilhelm, "Analysis of the rising incidence of thyroid cancer using the surveillance, epidemiology and end results national cancer data registry," Surgery, vol. 148, no. 6, pp. 1147-1153, 2010.

[2] G. Pellegriti, F. Frasca, C. Regalbuto, S. Squatrito, and R. Vigneri, "Worldwide increasing incidence of thyroid cancer: update on epidemiology and risk factors," Journal of Cancer Epidemiology, vol. 2013, Article ID 965212, 10 pages, 2013.

[3] L. Davies and H. G. Welch, "Current thyroid cancer trends in the United States," JAMA Otolaryngology-Head \& Neck Surgery, vol. 140, no. 4, pp. 317-322, 2014.

[4] S. Park, C.-M. Oh, H. Cho et al., "Association between screening and the thyroid cancer "epidemic" in South Korea: evidence from a nationwide study," BMJ, vol. 355, 2016.
[5] N. C. Hodgson, J. Button, and C. C. Solorzano, "Thyroid cancer: is the incidence still increasing?" Annals of Surgical Oncology, vol. 11, no. 12, pp. 1093-1097, 2004.

[6] L. Davies and H. G. Welch, "Increasing incidence of thyroid cancer in the United States, 1973-2002," JAMA, vol. 295, no. 18, pp. 2164-2167, 2006.

[7] D. R. Blankenship, E. Chin, and D. J. Terris, "Contemporary management of thyroid cancer," American Journal of Otolaryngology, vol. 26, no. 4, pp. 249-260, 2005.

[8] B. R. Haugen, E. K. Alexander, K. C. Bible et al., "2015 american thyroid association management guidelines for adult patients with thyroid nodules and differentiated thyroid cancer: the american thyroid association guidelines task force on thyroid nodules and differentiated thyroid cancer," Thyroid, vol. 26, no. 1, pp. 1-133, 2016.

[9] S. C. Clement, R. P. Peeters, C. M. Ronckers et al., "Intermediate and long-term adverse effects of radioiodine therapy for differentiated thyroid carcinoma-a systematic review," Cancer Treatment Reviews, vol. 41, no. 10, pp. 925-934, 2015.

[10] S. L. Lee, "Complications of radioactive iodine treatment of thyroid carcinoma," Journal of the National Comprehensive Cancer Network, vol. 8, no. 11, pp. 1277-1287, 2010.

[11] M. D. Moore, E. Postma, K. D. Gray et al., "Less is more: the impact of multidisciplinary thyroid conference on the treatment of well-differentiated thyroid carcinoma," World Journal of Surgery, vol. 42, no. 2, pp. 343-349, 2018.

[12] E. K. Alexander and P. R. Larsen, "Radioiodine for thyroid cancer - is less more?" New England Journal of Medicine, vol. 366, no. 18, pp. 1732-1733, 2012.

[13] H. O. Mäenpää, J. Heikkonen, L. Vaalavirta, M. Tenhunen, and H. Joensuu, "Low vs. high radioiodine activity to ablate the thyroid after thyroidectomy for cancer: a randomized study," PloS One, vol. 3, no. 4, 2008.

[14] R. M. Tuttle and M. M. Sabra, "Selective use of RAI for ablation and adjuvant therapy after total thyroidectomy for differentiated thyroid cancer: a practical approach to clinical decision making," Oral Oncology, vol. 49, no. 7, pp. 676-683, 2013.

[15] T. B. Ghachem, I. Yeddes, I. Meddeb et al., "A comparison of low versus high radioiodine administered activity in patients with low-risk differentiated thyroid cancer," European Archives of Oto-Rhino-Laryngology, vol. 274, no. 2, pp. 655-660, 2017.

[16] T. Pilli, E. Brianzoni, F. Capoccetti et al., "A comparison of $1850(50 \mathrm{mCi})$ and $3700 \mathrm{MBq}(100 \mathrm{mCi})$ 131-iodine administered doses for recombinant thyrotropin-stimulated postoperative thyroid remnant ablation in differentiated thyroid cancer," The Journal of Clinical Endocrinology \& Metabolism, vol. 92, no. 9, pp. 3542-3546, 2007.

[17] M. M. Sabra, R. K. Grewal, R. A. Ghossein, and R. M. Tuttle, "Higher administered activities of radioactive iodine are associated with less structural persistent response in older, but not younger, papillary thyroid cancer patients with lateral neck lymph node metastases," Thyroid, vol. 24, no. 7, pp. 1088-1095, 2014.

[18] M. J. Jeon, W. G. Kim, E. K. Jang et al., "Thyroglobulin level in fine-needle aspirates for preoperative diagnosis of cervical lymph node metastasis in patients with papillary thyroid carcinoma: two different cutoff values according to serum thyroglobulin level," Thyroid, vol. 25, no. 4, pp. 410-416, 2015.

[19] S. Hertz and A. Roberts, "Radioactive iodine in the study of thyroid physiology," Journal of the American Medical Association, vol. 131, no. 2, pp. 81-86, 1946. 
[20] H. J. Kim, N. K. Kim, J. H. Choi et al., "Radioactive iodine ablation does not prevent recurrences in patients with papillary thyroid microcarcinoma," Clinical Endocrinology, vol. 78, no. 4, pp. 614-620, 2013.

[21] H. W. Lin and N. Bhattacharyya, "Survival impact of treatment options for papillary microcarcinoma of the thyroid," The Laryngoscope, vol. 119, no. 10, pp. 1983-1987, 2009.

[22] E. Ruel, S. Thomas, M. Dinan, J. M. Perkins, S. A. Roman, and J. A. Sosa, "Adjuvant radioactive iodine therapy is associated with improved survival for patients with intermediate-risk papillary thyroid cancer," The Journal of Clinical Endocrinology \& Metabolism, vol. 100, no. 4, pp. 1529-1536, 2015.

[23] J. Jonklaas, N. J. Sarlis, D. Litofsky et al., "Outcomes of patients with differentiated thyroid carcinoma following initial therapy," Thyroid, vol. 16, no. 12, pp. 1229-1242, 2006.

[24] E. L. Mazzaferri and S. M. Jhiang, "Long-term impact of initial surgical and medical therapy on papillary and follicular thyroid cancer," The American Journal of Medicine, vol. 97, no. 5, pp. 418-428, 1994.

[25] A. M. Sawka, K. Thephamongkhol, M. Brouwers, L. Thabane, G. Browman, and H. C. Gerstein, "A systematic review and metaanalysis of the effectiveness of radioactive iodine remnant ablation for well-differentiated thyroid cancer," The Journal of Clinical Endocrinology \& Metabolism, vol. 89, no. 8, pp. 3668-3676, 2004.

[26] J. Jonklaas, D. S. Cooper, K. B. Ain et al., "Radioiodine therapy in patients with stage I differentiated thyroid cancer," Thyroid, vol. 20, no. 12, pp. 1423-1424, 2010.

[27] C. Schvartz, F. Bonnetain, S. Dabakuyo et al., "Impact on overall survival of radioactive iodine in low-risk differentiated thyroid cancer patients," The Journal of Clinical Endocrinology \& Metabolism, vol. 97, no. 5, pp. 1526-1535, 2012.

[28] M. G. Castagna, G. Cevenini, A. Theodoropoulou et al., "Post-surgical thyroid ablation with low or high radioiodine activities results in similar outcomes in intermediate risk differentiated thyroid cancer patients," European Journal of Endocrinology, vol. 169, no. 1, pp. 23-29, 2013.

[29] J. M. Han, W. G. Kim, T. Y. Kim et al., "Effects of low-dose and high-dose postoperative radioiodine therapy on the clinical outcome in patients with small differentiated thyroid cancer having microscopic extrathyroidal extension," Thyroid, vol. 24, no. 5, pp. 820-825, 2014.

[30] W. Cheng, C. Ma, H. Fu et al., "Low- or high-dose radioiodine remnant ablation for differentiated thyroid carcinoma: a meta-analysis," The Journal of Clinical Endocrinology \& Metabolism, vol. 98, no. 4, pp. 1353-1360, 2013.

[31] W. Sacks, C. H. Fung, J. T. Chang, A. Waxman, and G. D. Braunstein, "The effectiveness of radioactive iodine for treatment of low-risk thyroid cancer: a systematic analysis of the peer-reviewed literature from 1966 to April 2008," Thyroid, vol. 20, no. 11, pp. 1235-1245, 2010.

[32] J. Tu, S. Wang, Z. Huo, Y. Lin, X. Li, and S. Wang, "Recombinant human thyrotropin-aided versus thyroid hormone withdrawal-aided radioiodine treatment for differentiated thyroid cancer after total thyroidectomy: a metaanalysis," Radiotherapy and Oncology, vol. 110, no. 1, pp. 25-30, 2014.

[33] M. J. Hayat, N. Howlader, M. E. Reichman, and B. K. Edwards, "Cancer statistics, trends, and multiple primary cancer analyses from the surveillance, epidemiology, and end results (SEER) program," Oncologist, vol. 12, no. 1, pp. 20-37, 2007.

[34] I. F. Dingle, A. E. Mishoe, S. A. Nguyen, L. J. Overton, and M. B. Gillespie, "Salivary morbidity and quality of life following radioactive iodine for well-differentiated thyroid cancer," Otolaryngology-Head and Neck Surgery, vol. 148, no. 5, pp. 746-752, 2013.

[35] M. Wichers, E. Benz, H. Palmedo, H. J. Biersack, F. Grünwald, and D. Klingmüller, "Testicular function after radioiodine therapy for thyroid carcinoma," European Journal of Nuclear Medicine and Molecular Imaging, vol. 27, no. 5, pp. 503-507, 2000.

[36] C. Rubino, F. De Vathaire, M. E. Dottorini et al., "Second primary malignancies in thyroid cancer patients," British Journal of Cancer, vol. 89, no. 9, pp. 1638-1644, 2003. 